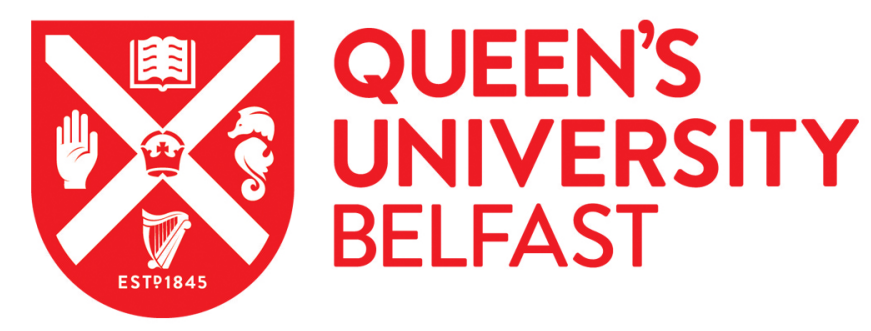

\title{
The need for enhanced psychological support in esophageal cancer- an exploratory study of the perception of HCPs, patients, and carers
}

Graham-Wisener, L., Collins, L., Hanna, J., \& Dempster, M. (2018). The need for enhanced psychological support in esophageal cancer-an exploratory study of the perception of HCPs, patients, and carers. Diseases of the Esophagus. https://doi.org/10.1093/dote/doy076

Published in:

Diseases of the Esophagus

Document Version:

Peer reviewed version

Queen's University Belfast - Research Portal:

Link to publication record in Queen's University Belfast Research Portal

Publisher rights

( 2018 The Authors. This work is made available online in accordance with the publisher's policies. Please refer to any applicable terms of use of the publisher.

\section{General rights}

Copyright for the publications made accessible via the Queen's University Belfast Research Portal is retained by the author(s) and / or other copyright owners and it is a condition of accessing these publications that users recognise and abide by the legal requirements associated with these rights.

Take down policy

The Research Portal is Queen's institutional repository that provides access to Queen's research output. Every effort has been made to ensure that content in the Research Portal does not infringe any person's rights, or applicable UK laws. If you discover content in the Research Portal that you believe breaches copyright or violates any law, please contact openaccess@qub.ac.uk. 


\title{
The Need for Enhanced Psychological Support in Esophageal Cancer- an exploratory study of the perception of HCPs, Patients and Carers
}

\author{
Lisa GRAHAM-WISENER ${ }^{1,2}$, Louise COLLINS ${ }^{3}$, Julie HANNA ${ }^{3}$, and Martin \\ DEMPSTER $^{2}$
}

\author{
${ }^{1}$ Marie Curie Hospice Belfast, Marie Curie, Belfast. \\ ${ }^{2}$ School of Psychology, Queen's University Belfast \\ ${ }^{3}$ Belfast Health and Social Care Trust
}

Word Count: 4988

Contribution of authors: Conception or design of the experiment(s), or collection and analysis or interpretation of data: all authors. Drafting the manuscript or revising its intellectual content: all authors. Approval of the final version of the submitted manuscript: all authors.

Short form title: Esophageal cancer psychological support

Corresponding author: Lisa Graham-Wisener, Marie Curie Hospice Belfast. 1a Kensington Road, Belfast BT5 6NF, Northern Ireland. Email;lisa.graham@mariecurie.org.uk. Telephone;+44(0)28 90882048. 


\begin{abstract}
Esophageal cancer patients and carers report significant levels of psychological distress. Despite this, only a small number of patients and carers engage with existing psychological services. This study aimed to explore the perception of esophageal cancer patients, carers and healthcare professionals (HCPs) of psychological distress and current provision of support, the need for and format of tailored support and barriers to patient/carer engagement. Semi-structured interviews were undertaken with $n=14$ esophageal cancer patients, carers and HCPs. Directed content analysis was utilised to code the transcripts according to preconceived categories as defined by study aim. Participants reported key periods for heightened distress around diagnosis and postsurgery on discharge from hospital. Provision of existing support was not felt to be adequate. A number of patient/carer barriers to engaging with support were identified in addition to HCP barriers. Participants perceived enhanced psychological support as a priority supportive care need with a number of recommendations for tailoring existing support services more adequately to the clinical population. With reports of heightened psychological morbidity yet with few esophageal cancer patients engaging with psychological services, there is an impetus to develop detailed care pathways to facilitate meeting this prioritized support need.
\end{abstract}

\title{
Keywords;
}

Esophageal cancer surgery; Esophagectomy; Esophageal cancers; Patient-

Centered Care; Emotional distress; Emotional Adjustment 


\section{Introduction}

A diagnosis of esophageal cancer and subsequent curative treatment results in a significant and sustained level of psychological morbidity for the patient and carer to longer term follow-up [1;2;3), comparable to that reported with head and neck cancer [4]. There is an impetus in policy driver documents [5] to provide timely psychological support to both esophageal cancer patients and their carers. Although clinical practice guidelines on the management of esophageal cancer have rarely cited management of psychological distress or given detail as to what this would constitute, there is increased recognition of psychological support as a clinical priority [6].

Few esophageal cancer patients access existing psychological support services [7]. It is therefore crucial to examine how psychological support may best be tailored to this population. Existing tailored supportive care interventions for esophageal cancer patients and carers have demonstrated some promise [8], though there are as yet no interventions which evidence a positive effect on psychological morbidity. This evidence is urgently needed to inform the best model of care.

The research to date in esophageal cancer has largely included quantitative prevalence studies. The aim of the current study is to provide a novel understanding through qualitative enquiry of the perception of esophageal cancer patients, carers and healthcare professionals (HCPs) in relation to i) experience of distress and current provision of psychological support, ii) the need for tailored psychological support, iii) barriers to engaging with psychological support and iv) the preferred format of a tailored psychological intervention.

\section{Materials and Methods}

\section{Participants and Procedures}

Esophageal cancer patients and carers were recruited from an Oesophageal Patient Association Northern Ireland meeting. Convenience sampling was used with broad eligibility criteria, including i) a past diagnosis of oesophageal cancer (curative pathway) or currently caring for a person with this diagnosis, and over 18 years of age. Health and 
social care professionals (HCPs) were recruited from a limited pool of clinicians and third sector workers at the single regional centre. Purposive sampling was used to ensure relevant HCPs from a range of disciplines across the treatment pathway (e.g. surgical, oncology). Inclusion criteria included adults over 18 years of age, who work as a health or social care professional within an upper GI cancer specialism or have a supportive input in the health and social care needs of this patient group.

The regional centre is aligned to a cancer support and information centre, where referrals may be made by the multidisciplinary team to a clinical psychology and counselling service. At time of interview, two Upper GI Clinical Nurse Specialists (CNS), total of 1 WTE, were aligned to the centre.

Semi-structured interviews, to allow issues of specific interest to the participant to be explored further, were conducted by LGW. Interviews were conducted face-to-face in either the patient/carer's home or university, or in the workplace environment in the case of the HCPs. The interviews lasted a mean of 18 minutes for patients and carers and a mean of 34 minutes for HCPs. The interview guide (see Appendix 1) was informed by Bowen and colleagues [9] proposed areas of focus to be addressed by feasibility studies. The guide was pilot tested with two members of clinical staff, no refinement needed.

\section{Analysis}

The interviews were audio-recorded and transcribed verbatim, with the anonymised transcripts subjected to directed content analysis [10], no software utilised. Coding was directed by the pre-conceived categories which formed the interview schedule (experience of distress and current provision, need for psychological support, barriers to engaging with support, format of intervention), with transcripts analysed for any instances of these categories. Instances of the categories within the interview schedule being identified were reviewed and agreed by both researchers. This approach to content analysis allows for detailed analysis of these aspects of the data which align to our specific research purpose [11]. 


\section{Results}

\section{Sample Characteristics}

The sample included 3 post-surgery esophageal cancer patients ( 1 female, 2 male, mean 45 months post-treatment end), and 2 female carers. The sample of $n=9$ stakeholders involved in providing health and social care to esophageal cancer patients included; $n=2$ AHP, $n=2$ medics, $n=4$ nurses, $n=1$ third sector representative. The sample had a mean of 10.75 years' experience of working directly in esophageal cancer and a mean of 18 years practice since initial qualification.

\section{Findings}

\section{Experience of distress and current provision of support}

All patient/carer participants described either experiencing heightened psychological distress themselves during the course of the patient's illness, or being acutely aware of the distress felt by others, "the stress end of it and the trauma end of it is as big an issue as the cancer itself" (P5-Carer).

A key point of heightened distress was perceived to be at the initial stages of the treatment pathway, with a lengthy staging process prior to a formal diagnosis marked by uncertainty. Patient/carer participants described the immediate shock of the formal diagnosis and the first few days of trying to "come to terms" with the information, continuing to feel "in a sort of vacuum" until the esophagectomy. Participants felt this was a missed opportunity for enhanced psychological support, "Nobody came near you. You were waiting for this big operation which you really didn't know that much about. You're told, this is major surgery that you're going to get, but underneath you don't know what major surgery means...I know I felt very isolated" (P3-Patient).

Esophageal cancer carers in the pre-operative period reported prioritising the wellbeing of the patient, often to their own detriment. Carers reported a desire for their own consultation, "if I'd have known the road ahead I maybe would have felt more prepared for what was going to happen" (P4-Carer). Carers experienced little control 
over the timing or content of information provided which often resulted in distress, with one participant describing at an early stage being told that the patient would "never be the same again" which was "as much a brutal piece of information as was being told that he had cancer" (P5-Carer).

HCP participants reported an awareness of heightened psychological distress from their respective contacts with esophageal cancer patients and carers across a complex illness trajectory, "esophageal cancer is a journey and there are plenty of cross roads along that journey that are very hard junctures for people to move on from" (P14-Support Worker).

All HCP participants agreed on the time period after surgery when the patient is discharged from hospital as being very distressing, with the patient leaving the "security blanket of what is the ward" (P12-Nurse). There is a contrast between the inpatient setting where "it's all planned out and there's that sequence of events" to the community where "they're having to deal with a lot" (P8-AHP). The impact of a "changed body", weight loss in the post-surgical period and hair loss from chemotherapy was believed to be distressing for patients. In particular were the sustained physical symptions post-surgery which were often experienced as unexpected and to impact on social functioning, "it's obviously mentioned the side effects of the surgery, but no one really takes that on. All they really want to know is that it is a cure for their cancer" (P8-AHP).

The role of the carer in the post-operative period is perceived as challenging, with carers involved in food preparation without necessarily having experience in this role. Participants discussed the "fraught" relationship between patient and carer, where "the carer wants to look after them and wants them to eat, but they don't feel like eating and it goes into a vicious circle" (P6-Nurse).

HCP participants felt patients and carers to hold expectations around a quick recovery post-surgery, resulting in heightened distress when this is not the case. Management of expectations and normalisation of a slow recovery is seen as key for HCPs at this time, "If you don't they will start to feel the pressure that they should be doing more than they are doing and that gets people down" (P7-Nurse). In general patients are perceived as being very fatigued during the post-operative period which can 
be de-motivating, "that's another thing from the cancer, they're just not able to do what they're used to and can get quite depressed" (P6-Nurse).

At longer term follow-up, HCP participants reported fear of recurrence to be common with patients experiencing distress around appointments and in respect of "every little symptom they have, they worry that it's a sign of something else going wrong in the body" (P8-AHP).

Patient/carer participants felt there was little psychological support available through specialist or primary care, though one participant self-referred to psychological services. Psychological support was considered to be embedded already in particular clinical roles. The CNSs were identified as undertaking a key role, "there was the support in that you could have spoken to the nurses about anything, they were approachable and very aware" (P2-Patient). Issues around resourcing were however highlighted, with the CNSs perceived to provide support only during active treatment and the initial postoperative period and not for longer term patients/carers where needed. More formalised contact with the CNS's was suggested.

Although the HCPs also perceived the CNS to undertake a key role in providing psychological support, there was a lack of clarity over the extent, "I know the specialist nurses are really good with them but how much, I don't know enough about their job to know how much follow-up they do" (P9-Nurse). Participants felt providing psychological support to be within a general HCP role, though were cognisant of their own limitations, “ Sometimes I think that is where I would love to have more training as you do as a person try to reassure people, but I probably haven't had much training on how to do that on a professional leve." (P8-AHP).

HCPs perceived psychological support to not be prioritised at an early stage in the treatment pathway, "I suppose we are very much on a treatment mode, an action mode, a diagnosis mode, and not necessarily thinking enough about the patient's psychological wellbeing" (P10-Medic). There was a noticeably dearth of services for patients after discharge, who "feel at sea" (P14-Support Worker) in terms of support services. There is acknowledgement of a lack of provision unless psychological distress is aligned to significant physical support need, "if people go home from hospital here there is no real 
provision at home for them. If there is nothing needing care physically, if people need support they really need to look for it themselves. This is the bottom line" (P7-Nurse). In particular the increasing number of elderly people living alone and without nearby family were thought to be at particular risk of distress.

HCP and patient/carer participants perceived patient peers to offer psychological support in both an informal setting and through patient associations. The role of peers in providing a shared identity and information on optimising symptom management in the post-operative period was highlighted, "I think networking, especially with our disease, is extremely good and necessary" (P3- Patient).

\section{Need for Psychological Support}

There was a clear consensus from the patient/carer participants that psychological support should be prioritised, with poor psychological wellbeing perceived as a predictor of physical recovery. All participants identified a role for more structured psychological support at diagnosis and discharge post-surgery. This was emphasised as a priority for both patient and carer, "Although it's very very hard for the patient and it's the one that it's happening to, the person on the sidelines just goes through it equally plus they have to watch somebody else suffer"(P4-Carer).

All HCP participants believed in the need for enhanced psychological support for this patient group, "I certainly think it would be very beneficial for a very high majority of patients and relatives" (P9-Nurse). Psychological support need was felt to currently be underacknowledged in care planning for esophageal cancer, "I do think it's really underestimated how hard it is" (P6-Nurse).

There was a clear sense from the HCPs of a distinct illness trajectory in esophageal cancer which necessitated tailored psychological support, "I just think cardiac surgery is the big thing, yet it's very much you come in, you get fixed, you get better. Yet this is ten times worse. It really is, you get worse before you get better" (P12-Nurse). The uniqueness of longer term effects from esophagectomy were considered key indicators for intervention, "So with other surgeries, people understand once you've had surgery 
you've recovered. Yes there is the chance of recurrence, but with esophageal cancer there are so many other issues around the surgery that are so debilitating. People don't appreciate it, so you really, really need the support to be able to get back to living with some sort of quality of mind and quality of life" (P14-Support Worker).

\section{Barriers to engaging with psychological support}

Patient/carer participants identified several barriers to engagement with psychological support. Public perception of psychological services were considered a stigma, "people don't want other people to know that they are seeing a psychologist, a psychiatrist, anything like that" (P1-Patient) alongside fear of being labelled with an additional diagnosis, "well it would be the fear factor of thinking 'I'm going nuts in the head as well' kind of thing" (P5-Carer). Participants suggested being mindful of the label given to the service, "Take the label off, labels conjure up all sorts of pictures and stereotypes. If you just say it's someone you can talk to” (P5-Carer). A potential facilitator was also suggested to be an opt-out system where all patients and carers are consistently offered psychological support "if it was part of the process where you spoke to somebody once you were diagnosed, I think that would be helpful. You can put on a front that oh we're going because we have to" (P4-Carer).

Individual differences with the patient or carer were suggested to impact on willingness to engage with psychological support, "Well if you're a shy person too who doesn't speak out much, you're probably just inclined to sit in your own corner. Not come out, and the older you get that's probably the case as you're less confident" (P3- Patient). There was an understanding that in dealing with an older, medically unwell population, psychological support needs to be as flexible as possible, "This disease in particular it is more elderly people who may not have their own transport, it is difficult for them to attend any type of appointment" (P1-Patient). Participants felt that the patient's symptoms after surgery may serve as a barrier to engagement, "I suppose people who have gone through the surgery, and maybe aren't too well, maybe frail, would be more resistant to go along to these meetings" (P3-Patient). 
Participants felt that encouraging patient and carer autonomy over access to psychological support could improve attendance, as patient and carer have less control across the rest of the treatment pathway, "Control is a big thing, if you feel that you are in control of this and what you do, that in itself gives the person back their own safety valves" (P5-Carer).

HCPs were also cognisant that there are barriers to patients/carers engaging with psychological support, "You can identify the distress, but when you look at the percentage of people who find psychological support in any form palatable, there's just a really big difference" (P13-AHP). This included an understanding that services are unlikely to be engaging with those most at need, "you'll get the people who don't really need it still coming forward" (P7-CNS).

HCPs reported practical barriers including geographical location, with the regional cancer centre requiring travel from across the province. Waiting lists after referral to psychological support were also seen as an "incredible barrier" to those who otherwise may have taken up the service (P13- AHP).

HCPs perceived hesitance in accessing psychological support to relate to the stigma of mental health services and perceived repercussions, "the idea of something psychological is what American teenage kids go to" (P10- Medic). Patients were not thought to perceive a distress response and psychological support as normative, "to say I think you need counselling, they automatically think they're abnormal. So it is difficult to bring up. You have to remember that it is natural to be distressed about what they're going through" (P7-Nurse). There was recognition that patients may not also "recognise what would be helpful to them" and that it would be the role of the HCP to help them be "aware of what their mind and body are doing, and then being aware that they can be helped" (P10-MedicParticipants felt patients may be hesitant to engage with psychological support as it is a new experience, "It's change, it's something new, and maybe they don't like that" (P12-Nurse).

A lack of information on available psychological services was perceived as a barrier, including a lack of awareness in HCPs of all services available to patients and carers and lack of a proactive approach in consistently providing this information. 
Esophageal cancer patients and carers were perceived to be "so overwhelmed with information" during active treatment that information on support services is often not attenuated to, "people often say that they weren't told that, and they definitely were. At that point they weren't ready and weren't able to take it on board" (P14-Support Worker).

\section{Format of enhanced psychological support}

Patient/carer participants felt that psychological support should be offered uniformly to all esophageal cancer patients and carers who would then have the autonomy to decide whether they needed the support. Participants were critical of clinicians relying on a screening tool to identify the most distressed individuals, and instead stressed the importance of making patients and carers aware of the support available at multiple timepoints, starting at an early stage in the treatment trajectory. The patients identified the role of a patient co-ordinator with broad knowledge of the constituent parts of the treatment pahway in facilitating access to psychological support.

Participants recommended enhanced psychological support to be available from early in the treatment trajectory and beyond active treatment, on a flexible basis, "It needs to be flexible, something that is rigid and denied is useless. Absolutely useless, better not there" (P5-Carer). It was stressed that early contact from the clinician delivering the support would be beneficial so the person is "known", but that support could be delivered at a distance (e.g. by telephone) after this point. Several participants felt a psychlogical intervention should be delivered by a person "completely independent" from their clinical team, with others suggesting this role to be aligned to the CNS, "I think initially there should be a counsellor of some sort. I think that's what they are putting (the CNSs) as" (P1-Patient). The CNS role was perceived as advantagous in fulfilling a dual role "They know the psychiatric side of it but they also know the medical side of the disease as well".

All HCP participants agreed on the need to tailor psychological support to fit flexibly around the complex illness trajectory experienced by esophageal cancer patients and their family. Although distress screening was considered neccessary under limited resources, participants also stressed the importance of patient autonomy in deciding to engage with support. All participants advocated for making patients aware of the 
intervention at an early stage in the treatment pathway, "if this is introduced from their initial diagnosis and they know, they maybe would then attend - normalising it" (P12Nurse). It was felt that an intervention should be presented as a routine option to patients who would be given several opportunities to access. HCP participants felt the surgeon, gastroenterologist or CNS should have key responsibility for identifying and referring patients/carers for support.

In relation to the format of enhanced psychological support, HCPs were supportive of a face to face intervention though acknowledged that attending sessions in person "is very difficult with the geography" and "time constraints" (P6-Nurse). A telephone intervention or video-conferencing were suggested, with the importance of meeting initially face to face emphasised, "I think from my profession, I find it very difficult to get a rapport with a patient if I am phoning them for the first time" (P8-AHP). Careful scheduling to ensure the patient or carer is able to have a confidential discussion was stressed if delivering at a distance. Although stressing potential benefits from peer support, an individual therapeutic approach was felt to be more lacking, "I would imagine someone working individually on the needs of that patient and the patient's carers may be more helpful" (P10-Medic).

Both HCP and patient/carer participants identified after diagnosis and immediately post-surgery to be the key points for intervention. HCP participants prioritised the post-surgical period and suggested scheduling psychological support alongside follow-up visits at the clinic. All participants agreed that support should be individually focused on the patient and carer, ideally with a dyadic element at a later stage to give opportunity to "hear the other's story" which was suggested to be "as therapeutic as anything you can give to them" (P10-Medic).

\section{Discussion}

Significant levels of anxious and depressive symptoms in esophageal cancer patients prior to surgery and in the first year post-treatment have been reported [3]. The 
current study is the first to contextualise these findings, with participants describing the initial shock of an often prolonged diagnosis, uncertainty during a complex and lengthy staging process prior to surgery, and a lack of preparation for life after surgery which includes a number of distressing factors; a slow recovery, significant physical side effects with various functional limitations and fear of recurrence.

Participants prioritised psychological distress as a supportive care need, mirroring previous research suggesting esophageal cancer patients to significantly value information on psychosocial aspects of care [12;13]. Provision of psychological support was however not felt to be adequate by either service users or HCPs. The opportunity for enhancing psychological support exists along the illness trajectory, though centers around two key periods; i) at diagnosis and ii) post-surgery at discharge from hospital.

There is recognition from the current study that psychological support exists within the role of various HCPs, yet there is a lack of training and confidence from HCPs in providing this support. NICE guidelines [14] recommend a staged approach to psychological support, with patients evidencing mild/moderate and temporal distress supported initially by HCPs directly involved in their care. With esophageal cancer patients and carers as a population also reporting high levels of distress which does not reach clinical cut-offs $[1 ; 2]$, enhancement on the therapeutic role of communication is one area for development [15].

The CNS role was reported as the key psychological support, though $\mathrm{HCP} /$ service user participants were frequently unaware of the particulars of the role. Despite the general increase in specialist nurses across the UK in recent years, Upper GI CNS's remain significantly under-resourced [16]. A focus on workforce planning appears key to ensure more structured and consistent contact with a CNS across the illness trajectory is achieved.

With few esophageal cancer patients to wishing to be referred for psychological support [7], it is important to be mindful of the reported barriers for engaging with existing psychological support which align to the broader cancer literature [17:18]. Findings also evidence the need to optimise psychological support of this patient population by tailoring the format and delivery of services to ensure they are acceptable. 
A number of considerations include developing a care pathway for the family carer, providing information on psychological support available at multiple points in the treatment pathway, and aligning psychological intervention more closely to existing appointments. Participants also reported scepticism over screening for psychological distress, instead favouring the provision of information on support available while emphasising patient/carer autonomy in engagement. The importance of expressed need has previously been recommended [19] as key in providing person-centred psychooncology services.

It is a limitation that the study focuses on the perspective of a relatively small number of patients, carers and HCPs from one cancer centre, limiting how generalisable the findings are. On the other hand this is a regional centre representing a high number of patients who are cared for from across a large geographical area. The current findings may usefully inform development of a survey to reach consensus on a UK level. Future research in this area may also adopt a longitudinal design to allow for a more direct understanding of need, provision and barriers/facilitators to accessing psychological services across the illness trajectory.

\section{Conclusion}

In conclusion, there is an evidenced need to optimise and tailor existing psychological support for esophageal cancer patients and carers. Key time points for intervention include after diagnosis, and in the initial post-surgical recovery period at discharge. A number of barriers have been identified for this patient group engaging with psychological support, along with recommendations which will usefully inform the development of detailed care pathways.

\section{Acknowledgements}

Many thanks to all participants for generously giving their time in support of this research. 


\section{References}

[1] Dempster M, McCorry NK, Brennan E, Donnelly M, Murray LJ, Johnston BT. Do changes in illness perceptions predict changes in psychological distress among oesophageal cancer survivors?. Journal of Health Psychology. 2011 Apr;16(3):500-9.

[2] Graham L, Dempster M, McCorry NK, Donnelly M, Johnston BT. Change in psychological distress in longer-term oesophageal cancer carers: are clusters of illness perception change a useful determinant?. Psycho-Oncology. 2016 Jun 1;25(6):663-9.

[3] Hellstadius Y, Lagergren J, Zylstra J, Gossage J, Davies A, Hultman CM, Lagergren P, Wikman A. A longitudinal assessment of psychological distress after oesophageal cancer surgery. Acta Oncologica. 2017 Jun 3;56(5):746-52.

[4] Hodges LJ, Humphris GM. Fear of recurrence and psychological distress in head and neck cancer patients and their carers. Psycho-Oncology. 2009 Aug 1;18(8):841-8.

[5] NHS Executive. Improving Outcomes Guidance in Upper Gastro-Intestinal Cancer. 2001. Available from; http://www.wales.nhs.uk/sites3/documents/362/UpperGIGuidance.pdf

[6] Lordick F, Mariette C, Haustermans K, Obermannová R, Arnold D. Oesophageal cancer: ESMO Clinical Practice Guidelines for diagnosis, treatment and follow-up. Annals of Oncology. 2016 Sep 1;27(suppl_5):v50-7.

[7] Jacobs M, Anderegg MC, Schoorlemmer A, Nieboer D, Steyerberg EW, Smets EM, Sprangers MA, Berge Henegouwen MI, Haes JC, Klinkenbijl JH. Patients with oesophageal cancer report elevated distress and problems yet do not have an explicit wish for referral prior to receiving their medical treatment plan. Psycho-Oncology. 2017 Apr $1 ; 26(4): 452-60$.

[8] Graham L, Wikman A. Toward improved survivorship: supportive care needs of esophageal cancer patients, a literature review. Diseases of the Esophagus. 2016 Dec 1;29(8):1081-9. 
[9] Bowen DJ, Kreuter M, Spring B, Cofta-Woerpel L, Linnan L, Weiner D, Bakken S, Kaplan CP, Squiers L, Fabrizio C, Fernandez M. How we design feasibility studies. American journal of preventive medicine. 2009 May 31;36(5):452-7.

[10] Hsieh HF, Shannon SE. Three approaches to qualitative content analysis. Qualitative health research. 2005 Nov;15(9):1277-88.

[11] Vaismoradi M, Turunen H, Bondas T. Content analysis and thematic analysis: Implications for conducting a qualitative descriptive study. Nursing \& health sciences. 2013 Sep 1;15(3):398-405.

[12] McNair AG, Brookes ST, Kinnersley P, Blazeby JM. What surgeons should tell patients with oesophago-gastric cancer: a cross sectional study of information needs. European Journal of Surgical Oncology (EJSO). 2013 Nov 30;39(11):1278-86.

[13] Graham-Wisener L, Dempster M. Peer advice giving from posttreatment to newly diagnosed esophageal cancer patients. Diseases of the Esophagus. 2017 Oct 1;30(10):17.

[14] National Institute for Health and Clinical Excellence. Improving supportive and palliative care for adults with cancer. 2017. NICE: London.

[15] Dean M, Street RL. A 3-stage model of patient-centered communication for addressing cancer patients' emotional distress. Patient education and counseling. 2014 Feb 28;94(2):143-8.

[16] Macmillan Cancer Support (2014b). Specialist adult cancer nurses in Northern Ireland. Accessed 14 March 2017 via; https:/www.macmillan.org.uk/ images/cnscensus-report-northern-ireland tcm9-283672.pdf

[17] Carolan CM, Smith A, Davies GR, Forbat L. Seeking, accepting and declining help for emotional distress in cancer: A systematic review and thematic synthesis of qualitative evidence. European journal of cancer care. 2017 Jun 8.

[18] Dilworth S, Higgins I, Parker V, Kelly B, Turner J. Patient and health professional's perceived barriers to the delivery of psychosocial care to adults with cancer: a systematic review. Psycho-Oncology. 2014 Jun 1;23(6):601-12.

[19] Faller H, Weis J, Koch U, Brähler E, Härter M, Keller M, Schulz H, Wegscheider K, Boehncke A, Hund B, Reuter K. Perceived need for psychosocial support depending on emotional distress and mental comorbidity in men and women with cancer. Journal of psychosomatic research. 2016 Feb 29;81:24-30. 


\section{Appendix 1. Interview Schedule with}

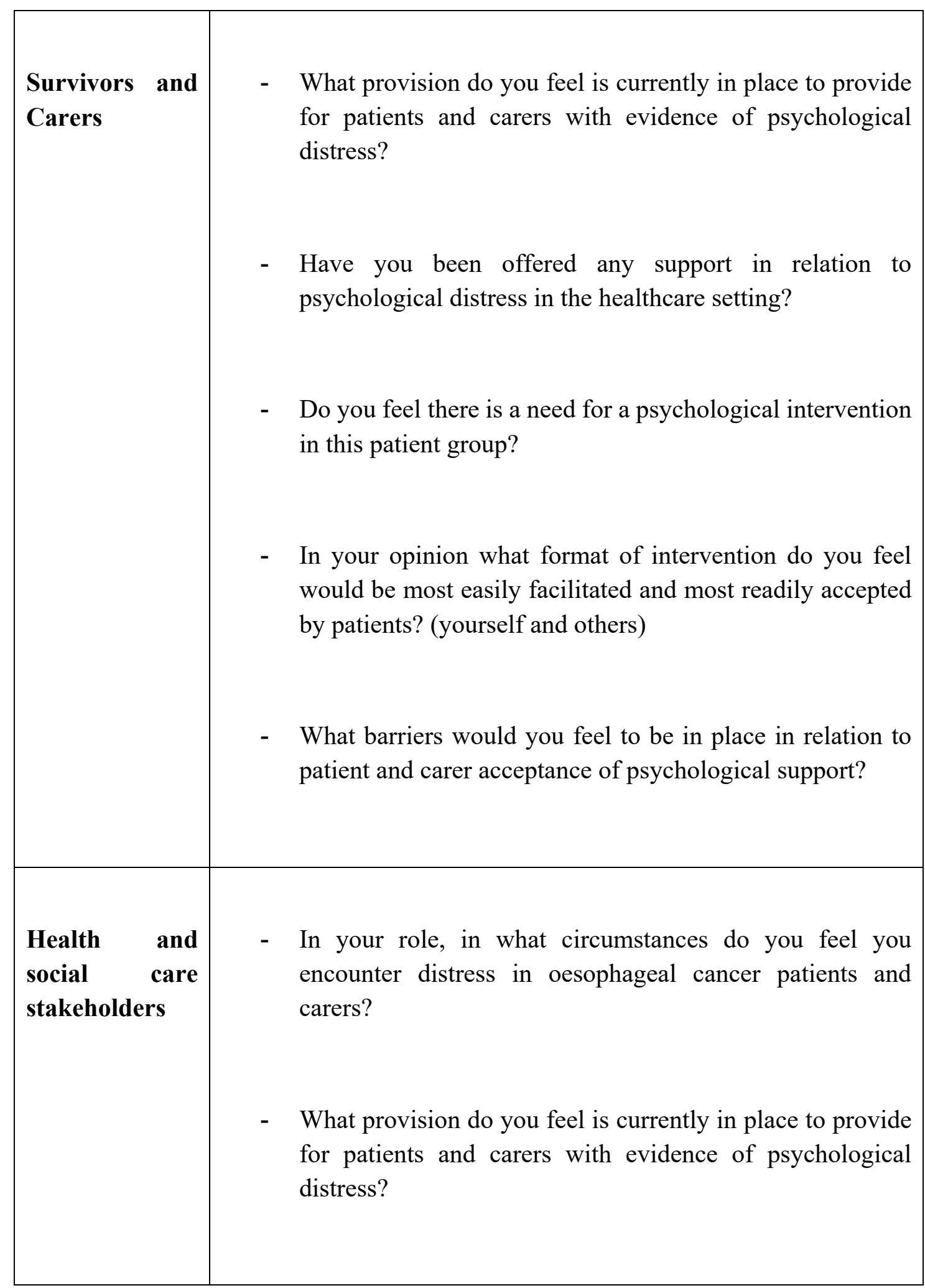




\begin{tabular}{|l|l|}
\hline & $\begin{array}{l}\text { Is there a specific referral pathway available for patients } \\
\text { with evidence of psychological distress? }\end{array}$ \\
- & $\begin{array}{l}\text { Do you feel there is need for a psychological intervention } \\
\text { in this patient group? }\end{array}$ \\
- & $\begin{array}{l}\text { In your opinion, what format of intervention do you feel } \\
\text { would be most easily facilitated and most readily accepted } \\
\text { by patients? }\end{array}$ \\
- & $\begin{array}{l}\text { What barriers do you feel may be in place in terms of } \\
\text { successfully implementing psychological support? For } \\
\text { patients? For staff? }\end{array}$ \\
\hline
\end{tabular}

